EPJ Web of Conferences 43, 01007 (2013)

DOI: $10.1051 /$ epjconf/20134301007

(C) Owned by the authors, published by EDP Sciences, 2013

\title{
Envelope loss of RGB/AGB stars and the formation of hot subdwarfs
}

\author{
Z. Han ${ }^{\mathrm{a}}$ and X. Chen
}

\author{
Key Laboratory for the Structure and Evolution of Celestial Objects, Yunnan Observatory of \\ the Chinese Academy of Sciences, Kunming 650011, China
}

\begin{abstract}
Low mass stars may lose their envelopes in the first giant branch (RGB) or the asymptotic giant branch (AGB) via envelope ejection (i.e. superwind). The envelope loss of AGB stars leads to the formation of carbon-oxygen (CO) white dwarfs (WDs), while the envelope loss of AGB stars may lead to the formation of helium WDs. We mainly focus here on where a RGB/AGB star loses its envelope during its evolution and we show the inital - final mass relation. We also propose a possible channel for the formation of single hot subdwarf stars, in which an old metal-rich RGB star with positive envelope binding energy may lose its envelope and the naked helium core gets ignited to become a hot subdwarf. We also review the wellestablished Han et al. scenario for the formation of hot subdwarf stars, in which binary interactions lead to the formation of both single and binary hot subdwarfs. By detailed binary evolution calculations, we show that PG 1018-047, a hot subdwarf binary with a main sequence companion and a very long orbital period of $756 \mathrm{~d}$, is explained naturally from the stable RLOF channel in the Han et al. scenario.
\end{abstract}

\section{ENVELOPE LOSS OF RGB/AGB STARS}

The evolution of a low- or intermediate-mass star can be briefly described as follows (see Fig. 1). The star spends most of its lifetime on the main sequence (MS) (point a to point b), burning hydrogen in its core. After hydrogen is exhausted in the core, the star expands and crosses the Hertzsprung gap quickly (points b to point $\mathrm{c}$ ) to settle on the base of the first giant branch (RGB) with a degenerate helium core and a convective envelope. Hydrogen burns in a shell on top of the helium core, the core grows in mass and the star climbs on the RGB (point $\mathrm{c}$ to point $\mathrm{d}$ ) until it ignites helium in its center and shrinks. The star spends a significant fraction of its lifetime (about 10 per cent) during the central helium burning phase (point $d$ to point e), and then ascends on the asymptotic giant branch (AGB), where the star has a degenerate carbon-oxygen $(\mathrm{CO})$ core surrounded by a helium burning shell and a hydrogen burning shell (point e to point $\mathrm{f}$ ). When the burning shells become thin enough, the burning is unstable and the star experiences thermal pulses (TP). At some point, a superwind commences and the TP-AGB star loses its envelope (point $\mathrm{f}$ ). After the envelope loss, the star is left with an exposed core and the temperature of the naked core increases. The lost material (i.e. the former envelope) is shaped by a fast wind from the central star (i.e. the naked core) and is ionized by the ultraviolet (UV) radiation emitted from the high-temperature central star, it then becomes a planetary nebula, one of the most beautiful objects in the Universe. After the nebula has dispersed, the central star becomes a white dwarf (WD) and evolves along the WD cooling track. The destiny of a low- or intermediate-mass star is normally a WD.

\footnotetext{
ae-mail: zhanwenhan@ynao.ac.cn

be-mail: cxf@ynao.ac.cn
}

This is an Open Access article distributed under the terms of the Creative Commons Attribution License 2.0, which permits unrestricted use, distribution, and reproduction in any medium, provided the original work is properly cited. 


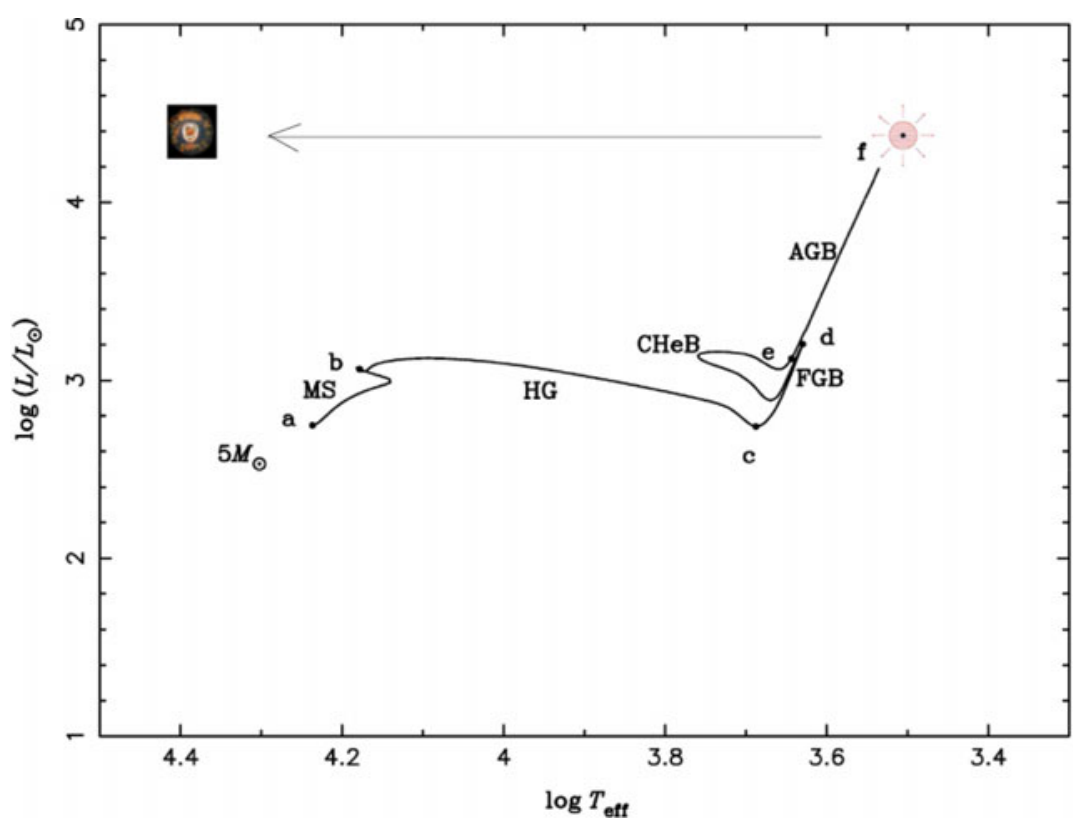

Figure 1. The evolution of a $5 M_{\odot}$ population I star in the HR diagram.

Exactly when an AGB star loses its envelope is crucial to our understanding of stellar evolution, and is also relevant to the chemical enrichment of interstellar material and to a better knowledge of type Ia supernovae. Following [1,2] assumed that the envelope of a giant star, either an AGB star or a RGB star, is lost when the envelope binding energy $\Delta W$ changes from negative to positive. The binding energy of the envelope is written as

$$
\Delta W=\int_{M_{\mathrm{c}}}^{M_{\mathrm{S}}}\left(-\frac{G m}{r}+U\right) \mathrm{d} m
$$

where $G$ is the gravitational constant, $m$ the mass at the local radius $r$, and $U$ is the internal energy of thermodynamics (involving terms due to ionization of $\mathrm{H}$ and dissociation of $\mathrm{H}_{2}$, as well as the basic $\frac{3 \Re T}{2 \mu}$ for a perfect gas). With such an assumption, [2] obtained initial - final mass relation for low- and intermediate-mass stars and the distribution of masses of the central stars of planetary nebulae. Both the relation and the distribution are supported observationally.

\section{INITIAL - FINAL MASS RELATIONS}

WDs are the endpoints of the evolution of low- and intermediate-mass stars, and are of significant importance to many fields of modern astrophysics. The relation between the initial mass $M_{\mathrm{i}}$ of a star and its final mass $M_{\mathrm{f}}$ (the WD mass) plays a key role in the use of WDs as cosmochronometers and in the understanding of the progenitors of type Ia supernovae (SNe Ia).

By calculating the envelope binding energy of stars, [2] obtained the initial - final mass relations for population I ( $Z=0.02)$ and population II $(Z=0.001)$ stars (see Section 1 and Fig. 2). The relations agree well with those derived observationally by [3]. [4] followed the evolution of AGB stars with mass ranging from 0.8 to $5 M_{\odot}$ through many accurately calculated thermal pulses and found an instability at the tip of the AGB, and that the instability or the thermal runaway is connected with the recombination of the partially ionized hydrogen. The initial - final relation they obtained is very similar to that of [2]. 


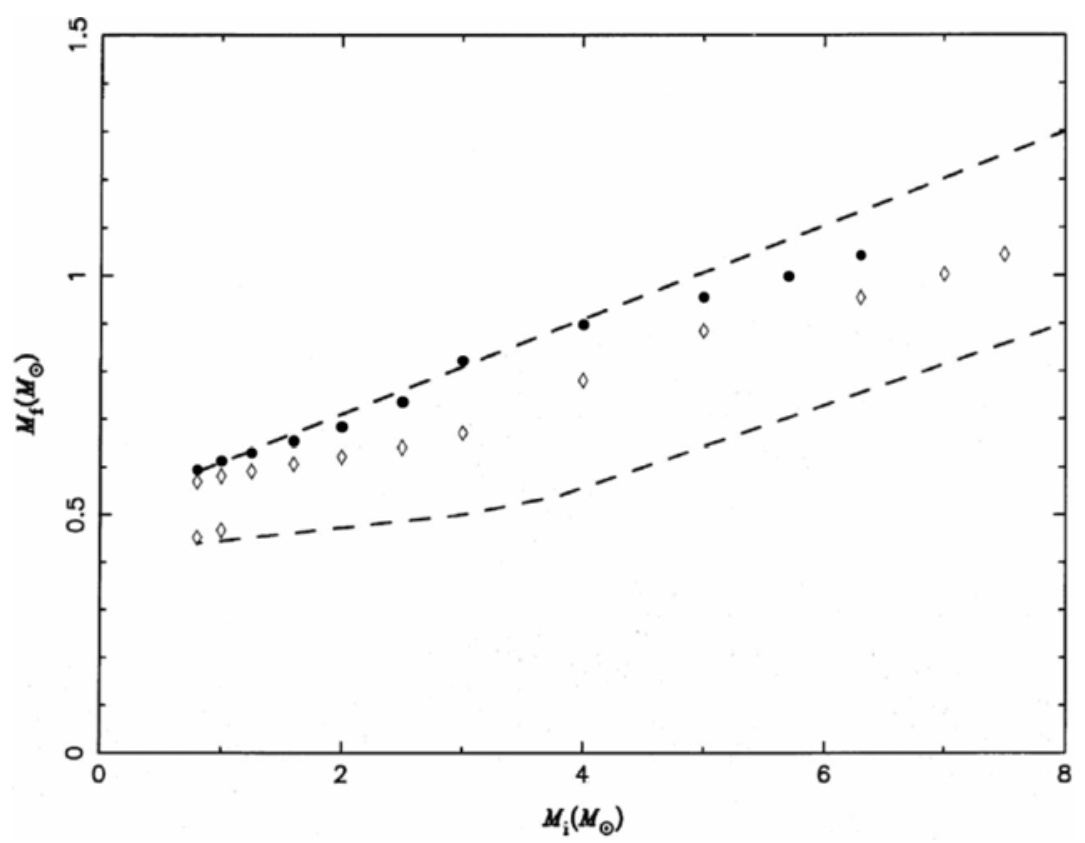

Figure 2. The initial - final mass relation of [2]. The filled circles represent population II stars while the diamonds represent population I stars. The region between the two dashed lines corresponds to the relation derived observationally by [3].

Adopting the same prescription, [5] extended the work of [2] to 12 metallicities ranging from extremely metal-poor $(Z=0.0001)$ to extremely metal-rich $(Z=0.1)$. The 12 metallicities are 0.0001 , $0.0003,0.001,0.004,0.01,0.02,0.03,0.04,0.05,0.06,0.08,0.1$, respectively. They found that the initial-final mass relation depends on metallicity. The final mass increases with $Z$ for $Z \geq 0.04$, and with decreasing $Z$ for $Z \leq 0.03$. [2] found that the envelope of a low-mass population I star (with a mass less than $\sim 1 M_{\odot}$ ) may get lost at RGB, and similarly, [5] showed that the envelope of low-mass stars with $Z \geq 0.02$ may get lost at RGB.

\section{HOT SUBDWARF STARS FROM VARIOUS CHANNELS}

Hot subdwarf stars are generally considered to be helium-core burning stars with extremely thin hydrogen envelopes $\left(<0.02 M_{\odot}\right)$ [6]. They have a typical mass of $\sim 0.5 M_{\odot}$ and are located between the upper main sequence and the white-dwarf sequence in the Hertzsprung-Russell (HR) diagram. As galactic field stars, they include subdwarf $\mathrm{O}(\mathrm{sdO})$ and $\mathrm{B}(\mathrm{sdB})$ stars. They are also found in globular clusters where they are known as extreme horizontal branch (EHB) stars. Hot subdwarf stars are important in the study of stellar evolution, stellar seismology, and structure and evolution of the Galaxy.Moreover, they are the major source of far-ultraviolet (far-UV) excess, i.e. the UV-upturn, of early-type galaxies and are a probe of the formation and evolution of elliptical galaxies.

\subsection{A possible envelope ejection channel}

In order to evolve to a hot subdwarf star, a RGB star needs to lose its envelope at the tip of the RGB and to get the remnant helium-core ignited. The loss of the envelope is not possible with a normal stellar wind, e.g. a Reimers wind, unless the wind is strongly enhanced. Observationally, more than half of hot 


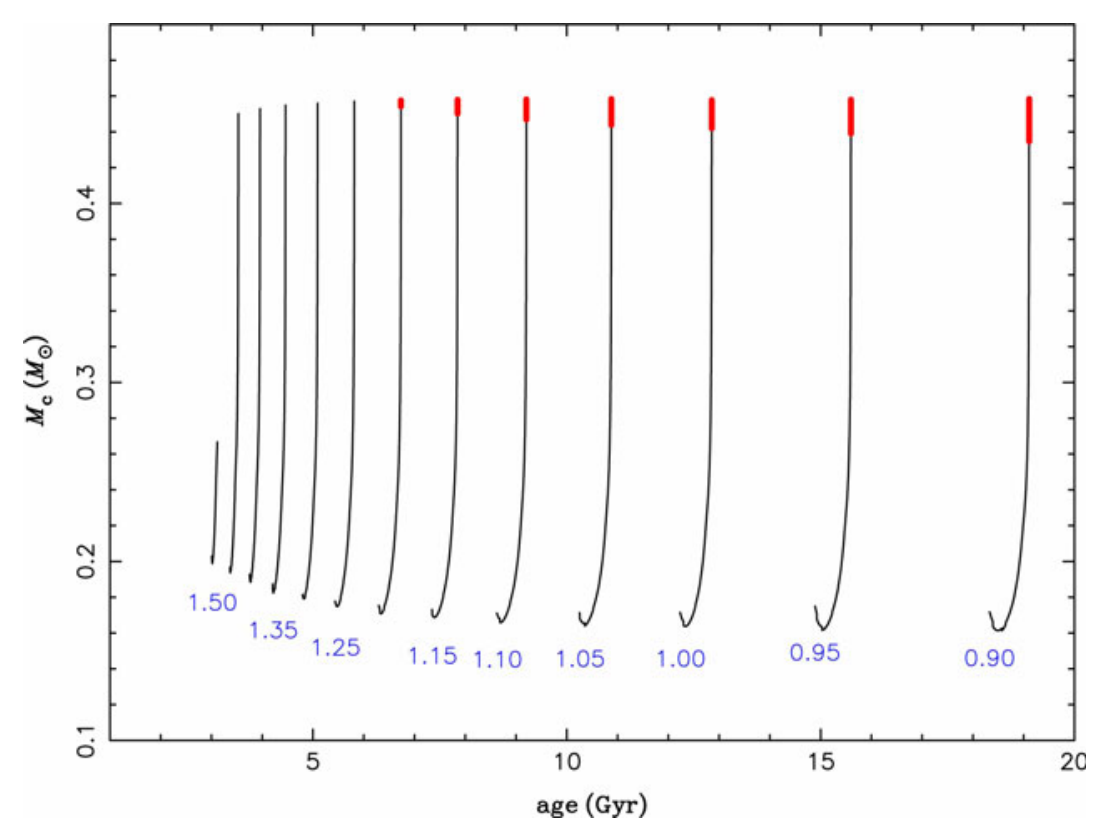

Figure 3. The core mass evolution with age for low mass RGB stars with a metallicity $Z=0.04$. The masses of the RGB stars are denoted at the bottom of each track, and the thick segments of the tracks denote the phase where the envelope binding energy is positive.

subdwarf stars are part of a binary system. [7-9] proposed a binary senario for the formation of both single and binary hot subdwarfs, and the scenario has successfully reproduced the observations.

Though the Han et al. scenario is successful, we should not ignore that, as shown in Sections 1 and 2, an old metal-rich star may have a positive envelope binding energy at the tip of the RGB and is speculated to lose (or "eject") its envelope to leave a naked helium core. The naked helium core may get ignited to become a hot subdwarf. We therefore calculated a series of evolutions of single stars. In our calculation, we adopted 8 metallicities, i.e. $Z=0.01,0.02,0.03,0.04,0.05,0.06,0.08,0.10$ (with $X=0.76-3 Z$ ). For each metallicity, we followed the evolution of single stars of masses $0.70,0.75$, $0.80, \ldots \ldots 1.45,1.50 M_{\odot}$. We found that a hot subdwarf star can be obtained this way in a Hubble time if the metallicity is larger than or equal to 0.02 . The higher the metallicity is, the more likely a hot subdwarf star is to be produced. Figure 3 shows, as an example, the evolution of low-mass RGB stars with a metallicity $Z=0.04$.

NGC 6791 is a metal-rich (with a metallicity of $[\mathrm{Fe} / \mathrm{H}] \sim+0.4$ ) and old (with an age of $\sim 8 \mathrm{Gyr}$ ) open cluster. It has the lowest binarity (see Sect. 3.2 below for the binary scenario of hot subdwarf formation) but shows an EHB [10]. We suspect that those EHB stars may form from the envelope ejection channel.

\subsection{The Han et al. scenario}

More than half of hot subdwarf stars are found in binaries, and [7-9] proposed a binary scenario to explain both binary and single hot subdwarf stars. In the scenario, there are three formation channels for hot subdwarf stars: stable Roche lobe overflow (RLOF) for hot subdwarf binaries with long orbital periods, common envelope (CE) ejection for hot subdwarf binaries with short orbital periods, and the merger of helium WDs to form single hot subdwarf stars. In the stable RLOF channel, the mass donor fills its Roche lobe near the tip of the RGB and experiences a stable mass transfer, then its envelope is 


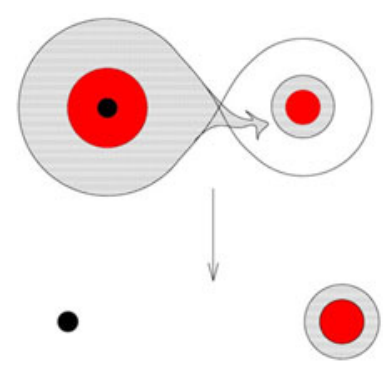

Figure 4. A schematic diagram for stable RLOF of a red giant binary system.

stripped off by the RLOF, and the naked helium core (with a thin hydrogen envelope) gets ignited to produce a hot subdwarf star. In the CE ejection channel, the mass donor fills its Roche lobe near the tip of the RGB which leads to a dynamically unstable mass transfer and to the formation of a CE. The CE ejection leaves a naked helium core (with a thin hydrogen envelope) and the naked helium core is ignited to produce a hot subdwarf star. In the merger channel, a close helium WD pair coalesces due to angular momentum loss via gravitational wave radiation, and the merged product ignites helium to become a hot subdwarf.

The binary scenario successfully explained the main observational characteristics of field hot subdwarf stars: their distributions in the orbital period-minimum companion mass diagram, and in the effective temperature $v s$ surface gravity diagram; their distributions of orbital period and mass function; their binary fraction and the fraction of hot subdwarf binaries with WD companions; their birth rates; and their space density. The model is indeed a step forward and is widely used in the study of hot subdwarf stars (e.g., [11]).

\section{PG 1018-047}

The Han et al. scenario produces single hot subdwarf stars via the merger of helium WD pairs, hot subdwarf binaries with short orbital periods via CE ejection, and hot subdwarf binaries with long orbital periods via stable RLOF. The scenario is well established, but the orbital periods of hot subdwarf binaries from the stable RLOF channel have not been well determined observationally due to the long orbital periods. [12] reported that the hot subdwarf binary PG 1018-047 has a MS companion and an orbital period of $760 \mathrm{~d}$, which is longer than the orbital period predicted by the stable RLOF channel of the Han et al. scenario (the predicted peak is $\sim 500 \mathrm{~d}$ ). This casts doubts on the stable RLOF channel.

Note, however, that the main interest of [7] and [8] was the CE channel, which is for hot subdwarf binaries with short orbital periods. The stable RLOF channel was treated rather crudely in their study. For a red giant binary with a primary mass $M_{1}$, a secondary mass $M_{2}$ and an orbital period $P_{\mathrm{i}}$, they assumed a final primary mass $M_{1 \mathrm{f}}$ (i.e. the hot subdwarf mass) after the RLOF, a mass loss fraction $\beta$ and an angular momentum loss $\mathrm{d} J$ during the stable RLOF, and then derived the final secondary mass $M_{2 \mathrm{f}}$, and the final orbital period $P_{\mathrm{f}}$ after the RLOF. From angular momentum point of view, it is obvious that a large $\mathrm{d} J$ would result in a smaller $P_{\mathrm{f}}\left(M_{1 \mathrm{f}}\right.$ is assumed to be independent of $\mathrm{d} J$ and $\left.P_{\mathrm{f}}\right)$. Strictly speaking, this is not quite right. For giant stars with degenerate cores, there exists a well known core mass - stellar radius $\left(M_{\mathrm{c}}-R\right)$ relation. During stable RLOF, the donor (the giant star) is Roche-lobe filling (see Fig. 4), a given $M_{\mathrm{c}}$ would correspond to a specific value of $R$, then to a specific value of $R_{\mathrm{L}}$, and therefore a specific value of orbital period $P$. In other words, there exists a $M_{\mathrm{c}}-P$ relation for a giant binary during RLOF phase. The RLOF stops when the envelope of the giant collapses, and we then have a $M_{\mathrm{f}}-P_{\mathrm{f}}$ relation. The relation does not depend on $\mathrm{d} J$. 


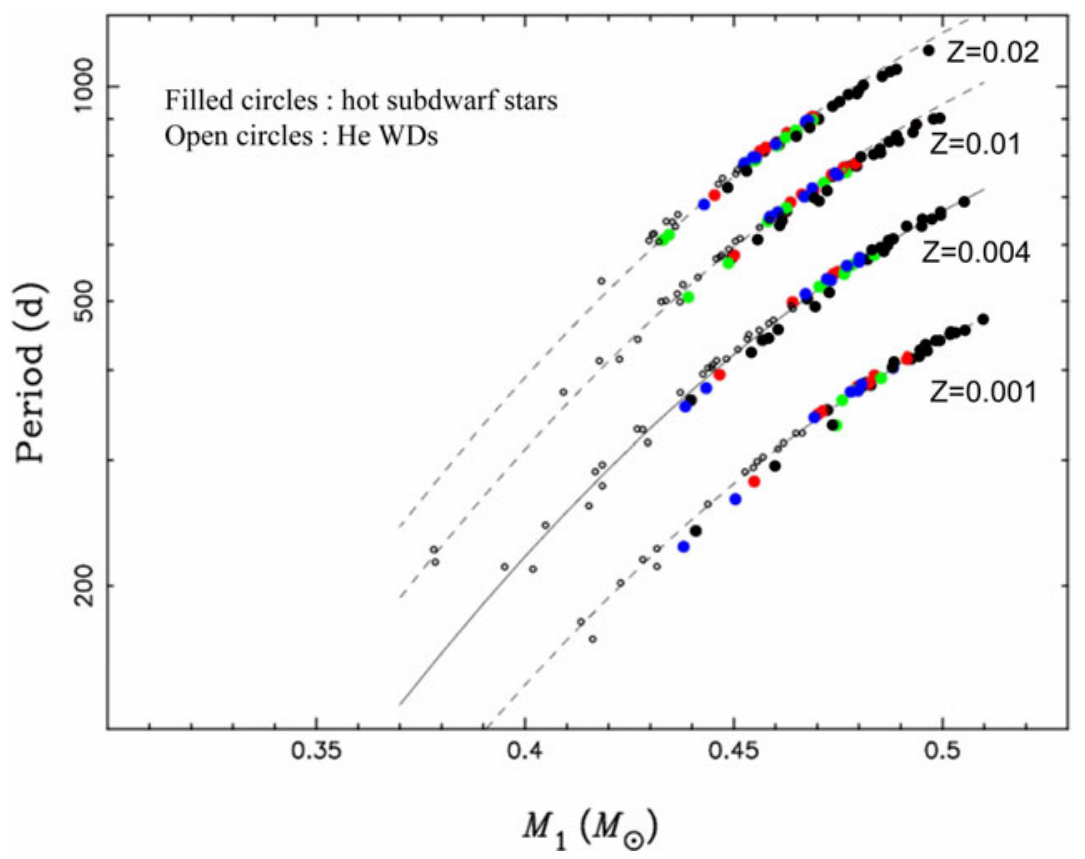

Figure 5. The result of stable RLOF from detailed evolution calculations of red giant binaries. The X-axis is for the remnant mass of the primary and the Y-axis for the orbital period after RLOF. Filled circles denote that the remnant of the primary is a hot subdwarf, while open circles denote that the remnant is a He WD. Four metallicities, i.e. $Z=0.02,0.01,0.004,0.001$, are adopted in the calculations.

In order to get a more realistic result from the stable RLOF channel, we carried out detailed binary evolution calculations. In these calculations, the mass of the primary and if the secondary $\left(M_{1 \mathrm{i}}, M_{2 \mathrm{i}}\right)$ (in $\left.M_{\odot}\right)$ are taken to be $(0.8,0.7),(1.0,0.9),(1.26,1.1),(1.60,1.5)$ alternatively, and various orbital periods have been chosen. We adopted various $\beta$, i.e., $0 ., 0.5,1.0$, and various angular momentum loss $\mathrm{d} J$ (i.e. the mass lost takes away the same specific angular momentum as pertains to the donor or to the companion). The calculations are made for 4 metallicities, i.e. $Z=0.02,0.01,0.004,0.001$. Figure 5 presents our result: it shows the hot subdwarf mass and the orbital period of the binaries resulting from the stable RLOF channel. From the figure, we see that stable RLOF does produce hot subdwarf binaries with orbital periods from $\sim 200$ to $\sim 1000 \mathrm{~d}$, and PG1018-047 is naturally explained.

We acknowledge the support from the NSFC (Nos. 11033008, 10973036), and the CAS (No. KJCX2-YW-T24 and the Talent Project of Western Light).

\section{References}

[1] Paczyńsk B., Ziółkowski J., Acta Astron., 18, (1968) 255

[2] Han Z., Podsiadlowski Ph., Eggleton P.P., MNRAS, 270, (1994) 121

[3] Weidemann V., Koester D., A\&A, 121, (1983) 77

[4] Wagenhuber J., Weiss A., A\&A, 290, (1994) 807

[5] Meng X., Chen X., Han Z., A\&A, 487, (2008) 625 
Ageing Low Mass Stars: From Red Giants to White Dwarfs

[6] Heber U., ARA\&A, 47, (2009) 211

[7] Han Z., Podsiadlowski P., Maxted P.F.L., Marsh T.R., Ivanova, N., MNRAS, 336 (2002), 449

[8] Han Z., Podsiadlowski P., Maxted P.F.L., Marsh T.R., MNRAS, 341, (2003) 669

[9] Han Z., A\&A, 484, (2008) L31

[10] Kalirai J., et al., ApJ, 671, (2007) 748

[11] O'Toole S.J., Heber U., Benjamin R.A., A\&A, 422, (2004) 1053

[12] Deca J., et al., MNRAS, 421, (2012) 2798 